УДК 517.95

\title{
The Inverse Problem for the Nonlinear Pseudoparabolic Equation of Filtration Type
}

\author{
Anna Sh. Lyubanova* \\ Institute of Space and Information Technologies \\ Siberian Federal University \\ Svobodny, 79, Krasnoyarsk, 660041
}

Russia

Received 25.06.2016, received in revised form 10.09.2016, accepted 20.11.2016

The paper discusses the correctness of the inverse problem on finding an unknown coefficient dependent on $t$ in the nonlinear pseudoparabolic equation of the third order with an additional information on the boundary. The existence and uniqueness theorem is proven. The proof of the theorem is carried out by the reduction of the original inverse problem to the equivalent one with an operator equation for the unknown coefficient.

Keywords: local existence and uniqueness theorem, a priori estimate, inverse problem, nonlinear higherorder equation, pseudoparabolic equation, filtration.

DOI: 10.17516/1997-1397-2017-10-1-4-15.

\section{Introduction}

This paper is devoted to the inverse problems of the identification of coefficients in the pseudoparabolic equation

$$
\left(u+L_{1} u\right)_{t}+L_{2} u=f,
$$

with the differential operators $L_{1}$ and $L_{2}$ of even order with respect to spacial variables. Such equations arises in the models of the heat transfer, filtration in the fissured media, quasistationary processes in the crystalline semiconductor (see more detailed review in [10, 11]).

The study of inverse problems for pseudoparabolic equations goes back to $1980 \mathrm{~s}$. The first result [9] refers to the inverse problems of determining a source function $f$ in $(0.1)$ with linear operators $L_{1}$ and $L_{2}$ of the second order, $L_{1}=L_{2}$. We should mention also the results in $[4,8]$ concerning with coefficient inverse problems for linear equation (0.1). In [8], the uniqueness theorem is obtained and an algorithm of determining a constant $a$ in the second order term is constructed. In [4], the solvability is established for two inverse problems of recovering the unknown coefficients in terms $u$ (the lowest term of $L_{2} u$ ) and $u_{t}$ of $(0.1)$.

In [7], the inverse problem of finding the coefficient $k$ depending on time in the equation

$$
(u+\eta M u)_{t}+k M u+g u=f,
$$

with integral data on the boundary is examined. Here $M$ is a second order linear differential operator in the space variables. Sufficient solvability and uniqueness conditions for a solution to the problem of identification of the coefficient $k(t)$ are established.

In the present article we establish solvability and uniqueness of solutions to the inverse problem of finding an unknown coefficient $k=k(t)$ in the nonlinear equation $(0.2)$ with the use of an additional information on the boundary (see (1.1)-(1.4)).

*lubanova@mail.ru

(c) Siberian Federal University. All rights reserved 


\section{Statement of the problem and preliminary results}

Let $\Omega$ be a bounded domain in $\mathbf{R}^{n}$ with a boundary $\partial \Omega \in C^{2}, T$ is an arbitrary real number and $Q_{T}=\Omega \times(0, T)$ a cylinder in $\mathbf{R}^{n+1}$ with the lateral boundary $S_{T}=\partial \Omega \times(0, T)$. The points of $\Omega$ are denoted by $x$, the points of $[0, T]$ by $t$, and the points of $Q_{T}$ by $(t, x)$. Below we use the following notation: $\|\cdot\|$ and $(\cdot, \cdot)$ are the norm and the inner product of $L^{2}(\Omega) ;\|\cdot\|_{j}$ and $\langle\cdot, \cdot\rangle_{j}$ are the norm of $W_{2}^{j}(\Omega)$ and the duality relation between $\mathscr{W}_{2}^{j}(\Omega)$ and $W_{2}^{-j}(\Omega)(j=1,2)$.

Let $M: W_{2}^{1}(\Omega) \rightarrow\left(W_{2}^{1}(\Omega)\right)^{*}$ is a linear differential operator of the form $M=-\operatorname{div}(\mathcal{M}(x) \nabla)+$ $m(x) I$ where $\mathcal{M}(x) \equiv\left(m_{i j}(x)\right)$ is a matrix of functions $m_{i j}(x), i, j=1,2, \ldots, n, m(x)$ is a scalar function and $I$ is the identity operator. We consider the following inverse problem. For a given constant $\eta$ and functions $f(t, x), \beta(t, x), U_{0}(x), \omega(t, x), \varphi_{1}(t), \varphi_{2}(t)$ find the pair of functions $(u(t, x), k(t))$ satisfying the equation

$$
(u+\eta M \psi(u))_{t}+k(t) M \psi(u)=f(t, x), \quad(t, x) \in Q_{T},
$$

the initial condition

$$
\left.(u+\eta M \psi(u))\right|_{t=0}=U_{0}(x), \quad x \in \Omega,
$$

the boundary data

$$
u=\beta(t, x), \quad(t, x) \in \bar{S}_{T},
$$

and the condition of overdetermination

$$
\int_{\partial \Omega}\left\{\eta \frac{\partial(\psi(u))_{t}}{\partial \bar{N}}+k(t) \frac{\partial \psi(u)}{\partial \bar{N}}\right\} \omega(t, x) d s+\varphi_{1}(t) k(t)=\varphi_{2}(t)
$$

for $t \in(0, T]$. Here $\frac{\partial}{\partial \bar{N}}=(\mathbf{n}, \mathcal{M}(x) \nabla)$ and $\mathbf{n}$ is the unit outward normal vector to $\partial \Omega$.

If $\omega(t, x) \equiv 1$ and $\varphi_{1} \equiv 0$, then the integral condition of overdetermination (1.4) means, for instance, the total discharge of a liquid through the surface of the ground.

We assume that the following conditions are fulfilled.

I. $M$ is an operator of elliptic type, that is, there exist positive constants $m_{1}$ and $m_{2}$ such that for any $v \in \stackrel{\circ}{W}_{2}^{1}(\Omega)$

$$
m_{1}\|v\|_{1}^{2} \leqslant\langle M v, v\rangle_{1} \leqslant m_{2}\|v\|_{1}^{2}
$$

and $m(x) \geqslant 0$ in $\Omega$.

II. $m_{i j}(x), \partial m_{i j} / \partial x_{l}, i, j, l=1,2, \ldots, n$, and $m(x)$ are bounded in $\Omega$ and the operator $M$ is selfadjoint, that is $m_{i j}(x)=m_{j i}(x)$ for $i, j=1,2, \ldots, n$.

III. The function $\psi(\rho)$ is an injection of $(-\infty,+\infty)$ onto itself. The injection is continuous and monotone, i. e.

$$
\left(\psi\left(\rho_{1}\right)-\psi\left(\rho_{2}\right)\right)\left(\rho_{1}-\rho_{2}\right) \geqslant 0
$$

for all $\rho_{1}, \rho_{2} \in(-\infty,+\infty), \rho_{1} \neq \rho_{2}$. The mapping $\psi^{-1}(v)$ from $L^{2}(\Omega)$ to $L^{q}(\Omega)(q \geqslant 2)$ is demicontinuous $\left(\psi^{-1}(\rho)\right.$ - the inverse of $\left.\psi(\rho)\right)$.

We denote by $a(t, x)$, and $b(t, x)$ the solutions of the problems

$$
\begin{array}{rll}
M \psi(a)=0 & \text { в } \Omega, & \left.a\right|_{\partial \Omega}=\beta(t, x), \\
M b=0 & \text { в } \Omega, & \left.b\right|_{\partial \Omega}=\omega(t, x),
\end{array}
$$

and introduce the additional notation:

$$
\begin{gathered}
\left\langle M v_{1}, v_{2}\right\rangle_{M}=\left(\mathcal{M}(x) \nabla v_{1}, \nabla v_{2}\right)+\left(m(x) v_{1}, v_{2}\right), \quad v_{1}, v_{2} \in W_{2}^{1}(\Omega) ; \\
\Psi(t)=\langle M \psi(a), b\rangle_{1, M}, \quad F(t, x)=a_{t}-f(t, x), \quad \bar{\Psi}=\max _{t \in[0, T]} \Psi(t), \quad \bar{\varphi}_{1}=\max _{t \in[0, T]} \varphi_{1}(t) .
\end{gathered}
$$


The existence and uniqueness of the solution to the problems (1.7) and

$$
\left.(u(0, x)+\eta M \psi(u(0, x)))\right|_{t=0}=U_{0}(x),\left.\quad u(0, x)\right|_{\partial \Omega}=\beta(0, x)
$$

are ensured by the Lemma $1.1[6]$. The trace $\left.u\right|_{t=0}=u_{0}$ belongs to $L^{p}(\Omega)$ and $\psi\left(u_{0}\right) \in W_{2}^{2}(\Omega)$. Moreover, the solutions of the problems (1.7) and (1.9) satisfy the comparison theorem (see Lemma $1.2[6])$.

By the solution of the direct problem (1.1)-(1.3) we mean the function $u$ of class $V=$ $\left\{v \mid \psi(v) \in C\left([0, T] ; W_{2}^{1}(\Omega)\right), v+\eta M \psi(v) \in C^{1}\left([0, T] ; W_{2}^{-1}(\Omega)\right)\right\}$ which satisfies the equation

$$
\left\langle(u+\eta M \psi(u))_{t}, v\right\rangle_{1}+\langle M \psi(u), v\rangle_{1}=\langle f, v\rangle_{1}
$$

for $\forall v \in L^{2}\left(0, T ; \stackrel{\circ}{W}_{2}^{1}(\Omega)\right)$, the identity $\left.\langle u+\eta M \psi(u)), v\right\rangle\left._{1}\right|_{t=0}=\left\langle U_{0}, v\right\rangle_{1}$ and the condition (1.3).

Lemma 1.1. Let the assumptions I-III be fulfilled, $\partial \Omega \subset C^{2}, k \in L^{\infty}(0, T), f \in C\left([0, T] ; L^{2}(\Omega)\right)$, $U_{0} \in L^{2}(\Omega), \psi(\beta) \in C^{1}\left([0, T] ; W_{2}^{1 / 2}(\partial \Omega)\right), \eta$ is a positive constant. Then the following assertions hold.

1) The direct problem (1.1)-(1.3) has a unique solution $u \in V$.

2) If in addition $\psi(\beta) \in C^{1}\left([0, T] ; W_{2}^{3 / 2}(\partial \Omega)\right)$ and for all $\rho \in(-\infty,+\infty)$ the inequality

$$
|\psi(\rho)| \geqslant c|\rho|^{p}
$$

holds with constants $c>0 u p \geqslant 1$, then $u \in W$ where $W=\left\{v \mid \psi(v) \in L^{\infty}\left(0, T ; W_{2}^{2}(\Omega)\right)\right.$, $\left.v \in L^{\infty}\left(0, T ; L^{2 p}(\Omega)\right),(v+\eta M \psi(v))_{t} \in L^{\infty}\left(0, T ; L^{2}(\Omega)\right)\right\}$.

Proof. 1) Let us define the function $\tilde{\psi}=\psi(u)-\psi(a)$ and rewrite the problem (1.1)-(1.3) in terms of $\tilde{\psi}$.

$$
\begin{aligned}
& {\left[\psi^{-1}(\tilde{\psi}+\psi(a))-a+\eta M \tilde{\psi}\right]_{t}+k(t) M \tilde{\psi}=F} \\
& {\left.\left[\psi^{-1}(\tilde{\psi}+\psi(a))-a+\eta M \tilde{\psi}\right]\right|_{t=0}=U_{0}-a(0, x),\left.\quad \tilde{\psi}\right|_{\partial \Omega}=0}
\end{aligned}
$$

In the hypotheses of the theorem the nonlinear operator $\tilde{M}$ mapping $L^{\infty}\left([0, T] ; W_{2}^{1}(\Omega)\right)$ into $L^{\infty}\left([0, T] ; W_{2}^{-1}(\Omega)\right)$ by the rule $\tilde{M} v=\psi^{-1}(v+\psi(a))-a+\eta M v$, is radially continuous and strongly monotone. Then, by Theorem 2.2 [3, Chapter 5], the problem (1.11)-(1.12) has a unique solution $\tilde{\psi} \in C\left([0, T] ; \stackrel{\circ}{W}_{2}^{1}(\Omega)\right)$ and $\tilde{M} \tilde{\psi} \in C^{1}\left([0, T] ; W_{2}^{-1}(\Omega)\right)$. Hence, $\psi(u) \in C\left([0, T] ; W_{2}^{1}(\Omega)\right)$ and $u+\eta M \psi(u) \in C^{1}\left([0, T] ; W_{2}^{-1}(\Omega)\right)$.

We estimate the norm of $\psi(u)$ in $C\left([0, T] ; W_{2}^{1}(\Omega)\right)$. In view of (1.11) and (1.12), the integration of (1.1) with respect to $t$ from 0 to $\tau, 0<\tau \leqslant T$ and the multiplication of the result by $\tilde{\psi}(u)$ in terms of the duality between $\dot{\circ}_{2}^{1}(\Omega)$ and $W_{2}^{-1}(\Omega)$ gives:

$$
(u-a, \tilde{\psi}(u))+\eta\langle M \tilde{\psi}(u), \tilde{\psi}(u)\rangle_{1}=\left\langle U_{0}-a_{0}+\int_{0}^{\tau}[F-k(t) M \tilde{\psi}(u)] d t, \tilde{\psi}(u)\right\rangle_{1}
$$

where $a_{0}(x)=a(0, x)$. Estimating the right term of (1.13) with the help of the Cauchy inequality, (1.5), (1.6) and applying the Gronwall lemma, one can obtain the inequality

$$
\|\tilde{\psi}(u)\|_{1}^{2} \leqslant \frac{1}{\eta^{2} m_{1}^{2}}\left[\left\|U_{0}-a_{0}\right\|_{W_{2}^{-1}(\Omega)}^{2}+T \int_{0}^{T}\|F\|_{W_{2}^{-1}(\Omega)}^{2} d t\right] \exp \left(\frac{m_{2}^{2} K^{2} T^{2}}{\eta^{2} m_{1}^{2}}\right) \equiv C_{1}
$$

$\left(K=\max _{t \in[0, T]}|k(t)|\right)$, whence

$$
\|\psi(u)\|_{1} \leqslant C_{1}^{1 / 2}+\|\psi(a)\|_{1}
$$


2) If (1.10) holds, then, by (1.15), we have

$$
\|u\|_{L^{2 p}(\Omega)} \leqslant \frac{1}{c}\left(C_{1}^{1 / 2}+\|\psi(a)\|\right) .
$$

Furthermore, integrating (1.1) with respect to $t$ from 0 to $\tau, 0<\tau \leqslant T$ and multiplying by $M \psi(u)$ in terms of the inner product of $L^{2}(\Omega)$ we reach the equality

$$
\eta\|M \psi(u)\|^{2}=\left(U_{0}-u, M \psi(u)\right)+\left(\int_{0}^{\tau} f d t, M \psi(u)\right)-\left(\int_{0}^{\tau} k(t) M \psi(u) d t, M \psi(u)\right) .
$$

In view of (1.16), (1.17) estimating the right term of this relation with the help of the Cauchy inequality and applying the Gronwall lemma to the resulting relation gives:

$$
\|M \psi(u)\| \leqslant \frac{\sqrt{2}}{\eta}\left((\operatorname{mes} \Omega)^{\frac{p-1}{2 p}}\|u\|_{L^{p}(\Omega)}+\left\|U_{0}\right\|+T \max _{t \in[0, T]}\|f\|\right) e^{\frac{K^{2} T}{2 \eta^{2}}} \equiv C_{2} .
$$

From (1.14), (1.18) and the inequality [5, Chapter 2]

$$
\|v\|_{2} \leqslant \chi\left(\|M v\|+\|v\|_{1}\right)
$$

for $v \in \stackrel{\circ}{W_{2}^{1}}(\Omega) \cap W_{2}^{2}(\Omega)$, where the constant $\chi$ depends on $n, m_{1}$, mes $\Omega$, we obtain the estimate

$$
\|\psi(u)\|_{2} \leqslant \chi\left(C_{2}+C_{1}^{1 / 2}\right)+\|\psi(a)\|_{2} \equiv C_{2}^{\prime} .
$$

(1.1) and (1.20) implies that $(u+\eta M \psi(u))_{t} \in L^{\infty}\left(0, T ; L^{2}(\Omega)\right)$.

Lemma 1.2. Let the assumptions I-III are fulfilled and the hypotheses of Lemma 1.1 providing the existence of the solution $u \in W$ to the direct problem (1.1)-(1.3) holds. Suppose that $k(t) \geqslant 0$ on $[0, T]$. Then the following assertions are valid.

1) If $f \geqslant 0$ almost everywhere in $Q_{T}, U_{0} \geqslant 0$ almost everywhere in $\Omega$ and $\beta \geqslant 0$ almost everywhere on $S_{T}$, then $u \geqslant 0$ for almost all $(t, x) \in Q_{T}$.

2) If $a_{t}-f \geqslant 0$ almost everywhere in $Q_{T}$ and $a(0, x)-U_{0} \geqslant 0$ almost everywhere in $\Omega$, then $u \leqslant$ a for almost all $(t, x) \in Q_{T}$.

Proof. 1) We multiply (1.1) by the function $\exp \left(\frac{1}{\eta} \int_{0}^{t} k(\tau) d \tau\right)$ and transfer the first summand to the right side of the obtained relation. Renaming $t$ as $\theta$ and integrating this relation with respect to $\theta$ from 0 to $t, 0<t \leqslant T$ and integrating by parts with respect to $\theta$ in the term contained $u_{t}$ we are led to the equality

$$
u+\eta M \psi(u)=U_{0} \exp \left(-\frac{1}{\eta} \int_{0}^{t} k d \tau\right)+\int_{0}^{t}\left(\frac{k}{\eta} u+f\right) \exp \left(-\frac{1}{\eta} \int_{\theta}^{t} k d \tau\right) d \theta .
$$

Let us consider the iterative scheme:

$$
\begin{gathered}
u^{i}+\eta M \psi\left(u^{i}\right)=U_{0} \exp \left(-\frac{1}{\eta} \int_{0}^{t} k d \tau\right)+\int_{0}^{t}\left(\frac{k}{\eta} u^{i-1}+f\right) \exp \left(-\frac{1}{\eta} \int_{\theta}^{t} k d \tau\right) d \theta \\
\left.u^{i}\right|_{S_{T}}=\beta(t, x), \quad i=1,2, \ldots ; \quad u^{0}=0 .
\end{gathered}
$$

Let $v^{i}$ and $v$ be the solutions of the equations $M v^{i}=u^{i}$ and $M v=u$, respectively, $\left.v^{i}\right|_{S_{T}}=$ $\left.v\right|_{S_{T}}=0$. We subtract (1.21) from (1.22) at the iteration $i$ and multiply the difference by $v^{i}-v$ in terms of the inner product of $L^{2}(\Omega)$. By (1.6), after integration by parts with respect to space variables and estimation of the right term in the resulting relation one can prove that vrai $\max _{t \in[0, T]}\left\|v^{i}-v\right\|_{1} \rightarrow 0$ and hence $u^{i} \rightarrow u$ in $L^{\infty}\left(0, T ; W_{2}^{-1}(\Omega)\right)$ as $i \rightarrow \infty$. Moreover, $u^{i}, u \in L^{2 p}\left(Q_{T}\right)$ by Lemma 1.1 , which implies that $u^{i} \rightarrow u$ weakly in $L^{2}\left(Q_{T}\right)$ as $i \rightarrow \infty$. In accordance with Lemma $1.2[6] u^{i} \geqslant 0, i=1,2, \ldots$. Then $u \geqslant 0$ for almost all $(t, x) \in Q_{T}$.

2) Let now the hypotheses of the second assertion hold. By the assertion 2) of Lemma 1.2 [6] $u^{i} \leqslant a$ for almost all $(t, x) \in Q_{T}, i=1,2, \ldots$. Since $u^{i} \rightarrow u$ weakly in $L^{2}\left(Q_{T}\right)$ as $i \rightarrow \infty$ and $a \in L^{2 p}\left(Q_{T}\right)$, this inequality holds for $u$ for almost all $(t, x) \in Q_{T}$. 


\section{The existence and uniqueness of the solution to the inverse problem}

From hereafter we suppose that the following condition on $\psi(\rho)$ is fulfilled.

IV. The function $\psi(\rho)$ is continuously differentiable on $(-\infty,+\infty)$. Moreover, $\psi^{\prime}(\rho) \geqslant \psi_{0}>0$ where $\psi_{0}$ is a constant and for every $\sigma>0$ there exists a constant $L(\sigma)>0$ such that for all $\rho_{1}, \rho_{2} \in[-\sigma, \sigma]$

$$
\left|\psi^{\prime}\left(\rho_{1}\right)-\psi^{\prime}\left(\rho_{2}\right)\right| \leqslant L(\sigma)\left|\rho_{1}-\rho_{2}\right| .
$$

By the solution of the inverse problem (1.1)-(1.4) we mean the pair of functions $\{u, k\}$ which belongs to the class $W\left(t^{*}\right) \times C\left(\left[0, t^{*}\right]\right)$, where $W\left(t^{*}\right)=\left\{v \mid \psi(v) \in C\left(\left[0, t^{*}\right] ; W_{2}^{2}(\Omega)\right), \quad v \in\right.$ $\left.C\left(\left[0, t^{*}\right] ; L^{4}(\Omega)\right), v_{t} \in L^{\infty}\left(0, t^{*} ; L^{2}(\Omega)\right),(\psi(v))_{t} \in L^{\infty}\left(0, t^{*} ; W_{2}^{2}(\Omega)\right)\right\}$ and satisfies the equation (1.1) for almost all $(t, x) \in Q_{T}$ and the conditions (1.2)-(1.4) for $t \in\left[0, t^{*}\right]$ where $0<t^{*} \leqslant T$.

Theorem 2.1. Let $n \leqslant 4$ and the assumptions $I-I V$ are fulfilled. Let also

(i) $\partial \Omega \subset C^{2}, f \in C\left([0, T] ; L^{2}(\Omega)\right), \psi(\beta) \in C^{1}\left([0, T] ; W_{2}^{3 / 2}(\partial \Omega) \cap L^{\infty}(\partial \Omega)\right), U_{0} \in L^{2}(\Omega)$, $\varphi_{1}, \varphi_{2} \in C([0, T]), \omega \in C\left([0, T] ; W_{2}^{3 / 2}(\partial \Omega)\right) ;$

(ii) $f \geqslant 0$ almost everywhere in $Q_{T}$ and $U_{0} \geqslant 0$ for almost all $x \in \Omega ; \beta$ and $\omega$ are nonnegative almost everywhere on $S_{T}$;

(iii) there is a number $\alpha>0$ such that

$$
\begin{gathered}
\varphi_{1}+\Psi \geqslant \alpha \\
a_{0}(x)-U_{0}(x) \geqslant 0 \\
F(t, x) \equiv a_{t}-f \geqslant 0 \\
\Phi \equiv \varphi_{2}+(f, b)-\eta\left\langle M(\psi(a))_{t}, b\right\rangle_{M} \geqslant \Phi_{0}+\frac{\alpha}{\alpha_{1}} \max _{t \in[0, T]}\left[\left(\left\|a_{t}\right\|+\frac{\|f\|}{\left(\psi_{0} \eta m_{1}\right)^{1 / 2}}\right)\|b\|\right],
\end{gathered}
$$

where $\Phi_{0}>0$ is a constant,

$$
\alpha_{1}=\alpha-\frac{2}{\left(\eta^{3} m_{1} \psi_{0}\right)^{1 / 2}}\left\|a_{0}-U_{0}\right\| \max _{t \in[0, T]}\|b\|>0 .
$$

Then there exists $t^{*}, 0<t^{*} \leqslant T$, such that the inverse problem (1.1)-(1.4) has a unique solution $\{u, k\} \in W\left(t^{*}\right)$. Moreover, the estimates

$$
0 \leqslant u \leqslant a, \quad 0 \leqslant k(t) \leqslant \delta^{*}(t)
$$

hold for $0 \leqslant t \leqslant t^{*}$ and $x \in \Omega$ where $\delta^{*}(t)$ is a continuous function on $\left[0, t^{*}\right]$.

Proof. We reduce the problem (1.1)-(1.4) to an equivalent inverse problem with a nonlinear operator equation for $k(t)$. To this end, we multiply (1.1) by the solution $b$ of the problem (1.8) in terms of the inner product in $L^{2}(\Omega)$ and integrate by parts with respect to $x$ at the second and third summands. By (1.4), this yields

$$
\left(u_{t}, b\right)-\varphi_{2}+\varphi_{1} k(t)+\eta \int_{\partial \Omega}(\psi(\beta))_{t} \frac{\partial b}{\partial \bar{N}} d s+k(t) \int_{\partial \Omega} \psi(\beta) \frac{\partial b}{\partial \bar{N}} d s=(f, b),
$$

whence in view of the fact that

$$
\int_{\partial \Omega}(\psi(\beta))_{t} \frac{\partial b}{\partial \bar{N}} d s=\left\langle M(\psi(a))_{t}, b\right\rangle_{M}, \quad \int_{\partial \Omega} \psi(\beta) \frac{\partial b}{\partial \bar{N}} d s=\langle M \psi(a), b\rangle_{M}=\Psi(t),
$$


we obtain the equation

$$
k(t)=\left[\Phi-\left(a_{t}, b\right)+\left((a-u)_{t}, b\right)\right]\left(\varphi_{1}+\Psi\right)^{-1} .
$$

Let a function $\delta(t)$ be positive and continuous on $[0, T]$. We define the cut-off function

$$
z_{\delta}(t)= \begin{cases}0, & z(t)<0, \\ z(t), & 0 \leqslant z(t) \leqslant \delta(t), \\ \delta(t), & z(t)>\delta(t)\end{cases}
$$

for every $z(t) \in L^{\infty}(0, T)$ and consider the following iterative scheme:

$$
\begin{gathered}
\left(u^{i}+\eta M \psi\left(u^{i}\right)\right)_{t}+k_{\delta}^{i-1}(t) M \psi\left(u^{i}\right)=f(t, x), \quad(t, x) \in Q_{T}, \\
\left.\left(u^{i}+\eta M \psi\left(u^{i}\right)\right)\right|_{t=0}=U_{0}(x), \quad x \in \Omega,\left.\quad u^{i}\right|_{S_{T}}=\beta(t, x), \\
k^{i}(t)=\left[\Phi-\left(a_{t}, b\right)+\left(\left(a-u^{i}\right)_{t}, b\right)\right]\left(\varphi_{1}+\Psi\right)^{-1}, \quad i=1,2, \ldots ; \quad k^{0}(t)=\delta(t) .
\end{gathered}
$$

By Lemma 1.2 and the assumption IV, the solution $u^{i}$ satisfies (2.7) and

$$
\psi(0) \leqslant \psi\left(u^{i}\right) \leqslant \psi(a)
$$

for every $i=1,2, \ldots$ Moreover, $u^{i}$ and $\psi\left(u^{i}\right)$ satisfy the estimates (1.14)-(1.16), (1.18) and (1.20) where $K=\max _{t \in[0, T]} \delta(t)$.

Let us now integrate (2.10) with respect to $t$ from 0 to $\tau, 0<\tau \leqslant T$, and multiply the result by $\psi_{u}^{i}=\psi\left(u^{i}\right)-\psi(a)$ in terms of the inner product of $L^{2}(\Omega)$. Integrating by parts in the second and third summands we obtain

$$
\left(u^{i}-a, \psi_{u}^{i}\right)+\left\langle\eta M\left(\psi_{u}^{i}\right)+\int_{0}^{\tau} k_{\delta}^{i-1} M\left(\psi_{u}^{i}\right) d t, \psi_{u}^{i}\right\rangle_{M}=\left(U_{0}-a_{0}-\int_{0}^{\tau} F d t, \psi_{u}^{i}\right) .
$$

Further, we multiply $(2.14)$ by $k_{\delta}^{i}(\tau)$ and integrate by $\tau$ from 0 to $\theta, 0<\theta<T$. Integrating by parts with respect to $\tau$ in the last term we are led to the equality

$$
\begin{gathered}
\int_{0}^{\theta} k_{\delta}^{i-1}\left[\left(u^{i}-a, \psi_{u}^{i}\right)+\eta\left\langle M \psi_{u}^{i}, \psi_{u}^{i}\right\rangle_{M}\right] d \tau+\frac{1}{2}\left\langle M \int_{0}^{\theta} k_{\delta}^{i-1} \psi_{u}^{i} d t, \int_{0}^{\theta} k_{\delta}^{i-1} \psi_{u}^{i} d t\right\rangle_{M} \\
=\left(-a_{0}+U_{0}-\int_{0}^{\theta} F d t, \int_{0}^{\theta} k_{\delta}^{i-1} \psi_{u}^{i} d t\right)+\int_{0}^{\theta}\left(F, \int_{0}^{\tau} k_{\delta}^{i-1}(t) \psi_{u}^{i} d t\right) d \tau .
\end{gathered}
$$

Estimating the right part of (2.15) with the use of (1.5) and the Gronwall lemma yields

$$
4 \eta \int_{0}^{\theta} k_{\delta}^{i-1}\left\langle M \psi_{u}^{i}, \psi_{u}^{i}\right\rangle_{M} d \tau+\left\langle M \int_{0}^{\theta} k_{\delta}^{i-1} \psi_{u}^{i} d t, \int_{0}^{\theta} k_{\delta}^{i-1} \psi_{u}^{i} d t\right\rangle_{M} \leqslant \frac{4\left(e^{\theta}-1\right)}{m_{1}} C_{3}^{2}(\theta),
$$

where $C_{3}=\left\|a_{0}-U_{0}\right\|+2 \theta \max _{t \in[0, T]}\|F\|$. By (1.5), (1.6), (2.14) and (2.16),

$$
\left\langle M\left(\psi_{u}^{i}, \psi_{u}^{i}\right\rangle_{M} \leqslant \frac{1}{\eta^{2} m_{1}} C_{3}^{2}(\tau)\left[2\left(e^{\tau}-1\right)^{1 / 2}+1\right]^{2} .\right.
$$

We now multiply $(2.10)$ by $\left(\psi_{u}^{i}\right)_{t}$ in terms of the inner product in $L^{2}(\Omega)$ and integrate by parts in the second and third summands of the resulting equation. This yields

$$
\left.\left(u_{t}^{i},\left(\psi\left(u^{i}\right)\right)_{t}\right)+\eta\left\langle M\left(\psi_{u}^{i}\right)_{t},\left(\psi_{u}^{i}\right)_{t}\right\rangle_{M}=-k_{\delta}^{i-1}\left\langle M\left(\psi_{u}^{i}\right),\left(\psi_{u}^{i}\right)_{t}\right\rangle_{M}+\left(u_{t}^{i},(\psi(a))_{t}\right)+\left(f,\left(\psi_{u}^{i}\right)_{t}\right)\right) .
$$


By estimating the right side of this equality with regard to (1.5), (2.17) and the assumption V, we obtain

$$
\psi_{0}\left\|u_{t}^{i}\right\|^{2}+\eta\left\langle M\left(\psi_{u}^{i}\right)_{t},\left(\psi_{u}^{i}\right)_{t}\right\rangle_{M} \leqslant \psi_{0}\left\{\left\|a_{t}\right\|^{2}+\frac{1}{\left(\eta m_{1} \psi_{0}\right)^{1 / 2}}\left[\|f\|+\frac{k_{\delta}^{i-1}(t)}{\eta} \tilde{C}_{3}(t)\right]\right\}^{2} .
$$

Here $\tilde{C}_{3}(t)=C_{3}(t)\left(2\left(e^{t}-1\right)^{1 / 2}+1\right)$.

We are now in a position to reach an estimate for $k^{i}(t)$. By (2.9), (2.12) and (2.18),

$$
\left[\Phi-\left(u_{t}^{i}, b\right)\right]\left(\varphi_{1}+\Psi\right)^{-1} \geqslant\left\{\Phi-\gamma(t)\|b\|-\delta(t) \frac{\tilde{C}_{3}(t)\|b\|}{\left(\eta^{3} m_{1} \psi_{0}\right)^{1 / 2}}\right\}\left(\varphi_{1}+\Psi\right)^{-1}
$$

where $\gamma(t)=\left(\left\|a_{t}\right\|+\left(\eta m_{1} \psi_{0}\right)^{-1 / 2}\|f\|\right)\|b\|$. In view of (2.5) the last expression is nonnegative when

$$
\delta(t) \leqslant \frac{(\Phi(t)-\gamma(t))\left(\eta^{3} m_{1} \psi_{0}\right)^{1 / 2}}{\tilde{C}_{3}(t)\|b\|} \equiv \delta^{\prime}(t) .
$$

On the other hand, by (2.6), there is $t_{1}, 0<t_{1} \leqslant T$, such that for $0 \leqslant t<t_{1}$

$$
\left[\Phi-\left(u_{t}^{i}, b\right)\right]\left(\varphi_{1}+\Psi\right)^{-1} \leqslant\left\{\Phi+\gamma(t)+\delta(t) \frac{C_{3}(t)\|b\|}{\left(\eta^{3} m_{1} \psi_{0}\right)^{1 / 2}}\left(2\left(e^{t}-1\right)^{1 / 2}+1\right)\right\} \alpha^{-1} \leqslant \delta(t) .
$$

In particular, $t_{1}$ can be chosen so that the inequality

$$
\varepsilon(t) \equiv \frac{2\left(e^{t}-1\right)^{1 / 2}}{\left(\eta^{3} m_{1} \psi_{0}\right)^{1 / 2}}\left[C_{3}(T)+T^{1 / 2} \max _{t \in[0, T]}\|F\|\right] \max _{t \in[0, T]}\|b\| \leqslant q \alpha_{1}
$$

holds on $\left[0, t_{1}\right]$ where $0<q<1$ is a constant which will be defined below. Then in view of $(2.5)$, $(2.6)$

$$
\alpha-\frac{C_{3}(t) \max _{t \in[0, T]}\|b\|}{\left(\eta^{3} m_{1} \psi_{0}\right)^{1 / 2}}\left(2\left(e^{t}-1\right)^{1 / 2}+1\right) \geqslant \alpha_{1}-\varepsilon(t) \geqslant(1-q) \alpha_{1}>0
$$

and

$$
\left[\Phi-\left(u_{t}^{i}, b\right)\right]\left(\varphi_{1}+\Psi\right)^{-1} \leqslant \delta(t)
$$

for

$$
\delta(t) \geqslant \frac{(\Phi+\gamma(t))\left(\eta^{3} m_{1} \psi_{0}\right)^{1 / 2}}{\alpha\left(\eta^{3} m_{1} \psi_{0}\right)^{1 / 2}-\tilde{C}_{3}(t)\|b\|} \equiv \delta^{\prime \prime}(t)
$$

on $\left[0, t_{1}\right]$. Moreover, $\delta^{\prime} \geqslant \delta^{\prime \prime}$ on an interval $\left[0, t^{*}\right], t^{*} \leqslant t_{1}$. Really, in view of $(2.5)$ and $(2.19)$ the inequality $\alpha_{1} \Phi(t)-\alpha \gamma(t) \geqslant \alpha_{1} \Phi_{0}$ holds on $\left[0, t_{1}\right]$. Therefore

$$
\delta^{\prime}-\delta^{\prime \prime} \geqslant\left(\eta^{3} m_{1} \psi_{0}\right)^{1 / 2}\left\{\alpha_{1} \Phi_{0}-2 \varepsilon(t) \bar{\Phi}\right\}\left\{\alpha_{1} \tilde{C}_{3}(T) \max _{t \in[0, T]}\|b\|\right\}^{-1},
$$

where $\bar{\Phi}=\max _{t \in[0, T]}|\Phi(t)|$. Taking $t^{*}$ from the condition

$$
\varepsilon\left(t^{*}\right) \leqslant \alpha_{1} \min \left\{\frac{\Phi_{0}}{2 \bar{\Phi}}, q\right\}
$$

we have $\delta^{\prime}-\delta^{\prime \prime} \geqslant 0$ for all $t \in\left[0, t^{*}\right]$. Thus, by $(2.20)$,

$$
0 \leqslant k^{i}(t) \leqslant \delta^{\prime \prime}(t), \quad i=1,2, \ldots
$$


for $t \in\left[0, t^{*}\right]$. Setting $\delta(t)=\delta^{\prime \prime}(t)$ in $(2.9)$ we conclude based on the last estimate that $k_{\delta}^{i}(t)$ coincides with $k^{i}(t)$ on $\left[0, t^{*}\right]$ for every $i=1,2, \ldots$.

Let us now turn to the estimation of $M \psi(u)$. Integrating (2.10) with respect to $t$ from 0 to $\tau$, $0<\tau \leqslant t^{*}$, and multiplying by $M \psi\left(u^{i}\right)$ in terms of the inner product in $L^{2}(\Omega)$ we are led to the equation

$$
\eta\|M \psi(u)\|^{2}+\left(\int_{0}^{\tau} k^{i-1} M \psi\left(u^{i}\right) d t, M \psi\left(u^{i}\right)\right)=\left(-u^{i}+U_{0}+\int_{0}^{\tau} f d t, M \psi\left(u^{i}\right)\right) .
$$

Multiplying this equality by $k^{i-1}$, integrating with respect to $\tau$ from 0 to $\theta$ and estimating the right term of the result with (1.16) we obtain

$$
\eta \int_{0}^{\theta} k^{i-1}\left\|M \psi\left(u^{i}\right)\right\|^{2} d \tau+\left\|\int_{0}^{\theta} k^{i-1} M \psi\left(u^{i}\right) d t\right\|^{2} \leqslant \frac{C_{4}^{2}}{\eta} \int_{0}^{\theta} k^{i-1} d \tau,
$$

where the constant $C_{4}>0$ depends on $\operatorname{mes} \Omega, p, c, C_{1}, T,\left\|U_{0}\right\|$ and $\max _{\theta \in[0, T]}\{\|\psi(a)\|,\|f\|\}$. Furthermore, rearranging the second term from the left side to the right one in (2.23) and estimating with (2.22) and (2.24), we arrive at the estimate

$$
\left\|M \psi\left(u^{i}\right)\right\| \leqslant \frac{C_{4}}{\eta^{3 / 2}}\left[\left(\int_{0}^{\theta} \delta^{\prime \prime}(\tau) d \tau\right)^{1 / 2}+1\right] .
$$

Let us define the operator $A: L^{\infty}\left(0, t^{*}\right) \rightarrow L^{\infty}\left(0, t^{*}\right)$ that takes each element $y(t) \in L^{\infty}\left(0, t^{*}\right)$ to the element

$$
A y(t)=\left[\varphi_{2}-\left(a_{t}-f, b\right)-\eta\left\langle M(\psi(a))_{t}, b\right\rangle_{M}+\left(\left(a-u_{y}\right)_{t}, b\right)\right]\left(\varphi_{1}+\Psi\right)^{-1},
$$

where $u_{y}$ is a solution of the problem (1.1)-(1.3) with $k(t)=y(t)$. In accordance with Lemma 1.1 the problem (1.1)-(1.3) has a unique solution $u_{y} \in L^{\infty}\left(0, t^{*} ; L^{2 p}(\Omega)\right)$ for every $y(t) \in L^{\infty}\left(0, t^{*}\right)$. Therefore the operator $A$ is well defined for each $y \in L^{\infty}\left(0, t^{*}\right)$. Moreover, by (2.22), the operator $A$ maps the set $Y\left(t^{*}\right)=\left\{y(t) \mid y(t) \in L^{\infty}\left(0, t^{*}\right), 0 \leqslant y(t) \leqslant \delta^{\prime \prime}\right\}$ into itself.

The equation (2.8) can be treated as the operator equation

$$
k=A k .
$$

In the hypotheses of the theorem $A$ is a contraction operator on $Y\left(t^{*}\right)$. Indeed, let $y_{1}(t), y_{2}(t) \in$ $Y\left(t^{*}\right)$ and $u_{y 1}, u_{y 2}$ be the solutions of the problem (1.1)-(1.3) with $k=y_{1}$ and $k=y_{2}$, respectively. The solutions $u_{y j}, j=1,2$, satisfy (2.16)-(2.18) with $y_{j}(t)$ instead of $k_{\delta}^{i}$. From (2.17) and assumption $\mathrm{V}$ it follows that

$$
\psi_{0}\left\|u_{y j}\right\|_{1} \leqslant\left\|\psi\left(u_{y j}\right)\right\|_{1} \leqslant \frac{\tilde{C}_{3}\left(t^{*}\right)}{\eta m_{1}}\left[2\left(e^{t^{*}}-1\right)^{1 / 2}+1\right]+\max _{t \in\left[0, t^{*}\right]}\|\psi(a)\|_{1} \equiv C_{5} .
$$

Let us consider the difference of the equations (1.1) for $\left\{u_{y 1}, y_{1}\right\}$ and $\left\{u_{y_{2}}, y_{2}\right\}$.

$$
\left[u_{y 1}-u_{y 2}+\eta M w_{y}\right]_{t}+y_{1} M w_{y}=\left(y_{2}-y_{1}\right) M \psi\left(u_{y 2}\right),
$$

where $w_{y} \equiv \psi\left(u_{y 1}\right)-\psi\left(u_{y 2}\right)$. By integrating this equality with respect to $t$ from 0 to $\tau, 0<\tau \leqslant t^{*}$, taking the inner product of the resultant relation by $w_{y}$ in $L^{2}(\Omega)$ and integrating by parts with respect to $x$, we obtain

$$
\left(u_{y 1}-u_{y 2}, w_{y}\right)+\left\langle\eta M\left(w_{y}+\int_{0}^{\tau} y_{1} w_{y} d \tau\right), w_{y}\right\rangle_{M}=\left\langle\int_{0}^{\tau}\left(y_{2}-y_{1}\right) M \psi\left(u_{y 2}\right) d t, w_{y}\right\rangle_{M}
$$


Let us multiply this relation by $y_{1}(\tau)$ and integrate with respect to $\tau$ from 0 to $\theta, 0<\theta \leqslant t^{*}$. By estimating the right side of the resultant equation with regard to (2.16) and (2.27), we are led to the inequality

$$
\eta \int_{0}^{\theta} y_{1}\left\langle M w_{y} w_{y}\right\rangle_{M} d \tau+\left\langle M \int_{0}^{\theta} y_{1} w_{y} d \tau, \int_{0}^{\theta} y_{1} w_{y} d \tau\right\rangle_{M} \leqslant C_{6}\left(\int_{0}^{\theta}\left|y_{2}-y_{1}\right| d t\right)^{2},
$$

where the constant $C_{6}>0$ depends on $\eta, m_{2}, t^{*}, C_{5}$ and $\max _{0 \leqslant \tau \leqslant t^{*}} \delta^{\prime \prime}(\tau)$ We now rearrange the last term of the left side to the right side of (2.29) and estimate the right side of the obtained equality with (2.27) and (2.30). By the assumption IV, we have

$$
2 \psi_{0}\left\|u_{y 1}-u_{y 2}\right\|^{2}+\eta\left\langle M w_{y}, w_{y}\right\rangle_{M} \leqslant \frac{1}{\eta}\left(C_{6}+m_{2} C_{5}\right)^{2}\left(\int_{0}^{\theta}\left|y_{2}-y_{1}\right| d t\right)^{2}
$$

Furthermore, we take the inner product of $(2.28)$ by $\left(w_{y}\right)_{t}$ in $L^{2}(\Omega)$, integrate by parts with respect to $x$ and rewrite the resultant equality as follows:

$$
\begin{gathered}
\left(\left(u_{y 1}-u_{y 2}\right)_{t}^{2}, \psi^{\prime}\left(u_{y 1}\right)\right)+\eta\left\langle M\left(w_{y}\right)_{t},\left(w_{y}\right)_{t}\right\rangle_{M}=-\left(\left(u_{y 1}-u_{y 2}\right)_{t},\left(w_{y}\right)_{t}\left(u_{y 2}\right)_{t}\right) \\
-y_{1}\left\langle M w_{y},\left(w_{y}\right)_{t}\right\rangle_{M}+\left(y_{2}-y_{1}\right)\left\langle M w_{y},\left(w_{y}\right)_{t}\right\rangle_{M} .
\end{gathered}
$$

Estimating the right side of (2.32) with regard to the fact that, by (2.1), (2.13), (2.18) and (2.31), the assumption IV and the embedding theorem,

$$
\begin{aligned}
& \left\|u_{y 1}-u_{y 2}\right\|_{L^{4}(\Omega)} \leqslant \frac{\kappa}{m_{1}^{1 / 2} \psi_{0}}\left\langle M w_{y}, w_{y}\right\rangle_{M}^{1 / 2} \leqslant \frac{\kappa\left(C_{6}+m_{2} C_{5}\right)}{\eta m_{1}^{1 / 2} \psi_{0}} \int_{0}^{\theta}\left|y_{2}-y_{1}\right| d t \\
& \psi_{0}^{1 / 2}\left\|\left(u_{y 2}\right)_{t}\right\|_{L^{4}(\Omega)} \leqslant\left\|\psi^{\prime}\left(u_{y 2}\right)\left(u_{y 2}\right)_{t}\right\|_{L^{4}(\Omega)} \leqslant \max _{t \in\left[0, t^{*}\right]}\left\{\left\|(\psi(a))_{t}\right\|_{L^{4}(\Omega)}+\psi_{0}^{1 / 2}\left\|a_{t}\right\|\right\}+ \\
& +\frac{1}{\left(\eta m_{1}\right)^{1 / 2}} \max _{t \in\left[0, t^{*}\right]}\left[\|f\|+\frac{\delta^{\prime \prime}}{\eta} C_{3}\left(t^{*}\right)\left(2\left(e^{t^{*}}-1\right)^{1 / 2}+1\right)\right] \equiv C_{7}
\end{aligned}
$$

we are led to the relation

$$
\psi_{0}\left\|\left(u_{y 1}-u_{y 2}\right)_{t}\right\|^{2}+\eta\left\langle M\left(w_{y}\right)_{t},\left(w_{y}\right)_{t}\right\rangle_{M} \leqslant\left[C_{8} \int_{0}^{t}\left|y_{2}-y_{1}\right| d \tau+C_{8}^{\prime}\left|y_{2}-y_{1}\right|\right]^{2},
$$

where $\kappa$ is the constant from the inequality of embedding $W_{2}^{1}(\Omega)$ into $L^{4}(\Omega)$, the positive constant $C_{8}$ depends on $m_{i}, i=1,2, \psi_{0}, \eta, \kappa, C_{5}, C_{6}, C_{7}$ and $\max _{t \in\left[0, t^{*}\right]} \delta^{\prime \prime} ; C_{8}^{\prime}=\left(\eta^{3} m_{1}\right)^{-1 / 2} C_{3}\left(t^{*}\right)\left(2\left(e^{t^{*}}-\right.\right.$ $\left.1)^{1 / 2}+1\right)$.

On the other hand, according to the definition of the operator $A$

$$
A y_{1}-A y_{2}=\left(\left(u_{y 1}\right)_{t}-\left(u_{y 1}\right)_{t}, b\right)\left(\varphi_{1}+\Psi\right)^{-1} .
$$

By (2.2), (2.7), (2.21) and (2.33), one can show that

$$
\left|A y_{1}-A y_{2}\right| \leqslant \frac{\|b\|}{\alpha}\left\|\left(u_{y 1}\right)_{t}-\left(u_{y 1}\right)_{t}\right\| \leqslant \frac{\|b\|}{\alpha \psi_{0}^{1 / 2}} C_{8} \int_{0}^{t}\left|y_{2}-y_{1}\right| d \tau+\left\{\frac{1}{2}+\frac{\varepsilon\left(t^{*}\right)}{\alpha}\right\}\left|y_{2}-y_{1}\right|
$$

on $\left[0, t^{*}\right]$. By $(2.21)$, we conclude that if $q<\min \left\{1, \frac{\alpha}{2 \alpha_{1}}\right\}$, then $q_{1} \equiv\left\{\frac{1}{2}+\frac{\varepsilon\left(t^{*}\right)}{\alpha}\right\}<1$ and

$$
\left|A y_{1}-A y_{2}\right| \leqslant \frac{\max _{t \in[0, T]}\|b\|}{\alpha \psi_{0}^{1 / 2}} C_{8} \int_{0}^{t}\left|y_{2}-y_{1}\right| d \tau+q_{1}\left|y_{2}-y_{1}\right|
$$


Introducing the equivalent norm $|\cdot|_{\mu}=$ vrai $\max _{t \in\left(0, t^{*}\right)}\left\{|\cdot| e^{-\mu t}\right\}$ in $L^{\infty}\left(0, t^{*}\right)$, we obtain the inequality

$$
\left|A y_{1}-A y_{2}\right|_{\mu} \leqslant\left(\frac{C_{8} \max _{t \in[0, T]}\|b\|}{\alpha \psi_{0}^{1 / 2} \mu}+q_{1}\right)\left|y_{2}-y_{1}\right|_{\mu}
$$

from $(2.34)$. If

$$
\frac{C_{8} \max _{t \in[0, T]}\|b\|}{\alpha \psi_{0}^{1 / 2} \mu}+q_{1}<1
$$

then $A$ is a contraction operator in the sense of the norm $|\cdot|_{\mu}$ on $Y\left(t^{*}\right)$. Hence by the contraction mapping principle the operator $A$ has a unique fixed point in $Y\left(t^{*}\right)$.

Let us come back to the iterative scheme (2.10)-(2.12). As mentioned above, $k_{\delta}^{i}(t)=k^{i}(t)$ on $\left[0, t^{*}\right]$ for every $i=0,1,2, \ldots$ By $(2.22)$ and $(2.35)$

$$
\left|A k^{i}-A k^{i-1}\right|_{\mu} \leqslant\left(\frac{\max _{t \in[0, T]}\|b\| \mid}{\alpha \psi_{0}^{1 / 2}} \frac{C_{8}}{\mu}+q_{1}\right)\left|k^{i}-k^{i-1}\right|_{\mu},
$$

which implies by $(2.36)$ that the sequence $k^{i}$ converges in sense of the norm of $L^{\infty}\left(0, t^{*}\right)$ as $i \rightarrow \infty$, its limit $k^{*}(t)$ is the solution of the equation (2.26) and satisfies the estimate (2.22).

Let $u^{*}$ is the solution of the problem (1.1)-(1.3) with $k=k^{*}$. Applying the arguments proved the estimates (2.31) and (2.33) to the difference of the equations (2.10) and (1.1) with $k=k^{*}$

$$
\left[u^{i}-u^{*}+\eta M\left(\psi\left(u^{i}\right)-\psi\left(u^{*}\right)\right)\right]_{t}+k^{*} M\left(\psi\left(u^{i}\right)-\psi\left(u^{*}\right)\right)=\left(k^{*}-k^{i}\right) M \psi\left(u^{i}\right)
$$

one can obtain the inequalities

$$
\begin{aligned}
& \left.2 \psi_{0}\left\|u^{i}-u^{*}\right\|^{2}+\eta \| \psi\left(u^{i}\right)-\psi\left(u^{*}\right)\right) \|_{1}^{2} \leqslant \frac{1}{\eta}\left(\left(C_{6}+m_{2} C_{5}\right) \int_{0}^{\theta}\left|k^{*}-k^{i}\right| d t\right)^{2}, \\
& \psi_{0}\left\|\left(u^{i}-u^{*}\right)_{t}\right\|^{2}+\eta m_{1}\left\|\left(\psi\left(u^{i}\right)-\psi\left(u^{*}\right)\right)_{t}\right\|_{1}^{2} \leqslant 2\left[C_{8} \int_{0}^{t}\left|k^{*}-k^{i}\right| d \tau+C_{8}^{\prime}\left|k^{*}-k^{i}\right|\right]^{2} .
\end{aligned}
$$

From these relations it follows that $u^{i} \rightarrow u^{*}$ in $C\left(\left[0, t^{*}\right] ; L^{2}(\Omega)\right) ; \psi\left(u^{i}\right) \rightarrow \psi\left(u^{*}\right)$ in $C\left(\left[0, t^{*}\right] ; W_{2}^{1}(\Omega)\right)$ and $u_{t}^{i} \rightarrow u_{t}^{*}$ in $L^{\infty}\left(0, t^{*} ; L^{2}(\Omega)\right)$ as $i \rightarrow \infty$. Moreover, the estimates $(2.7)$, (2.17) and (2.18) for $u^{*}$ and $u_{t}^{*}$ holds.

Let us integrate (2.37) with respect to $t$ from 0 to $\tau, 0<\tau \leqslant t^{*}$, take the inner product of the resultant equality by $M \psi^{i}=M\left(\psi\left(u^{i}\right)-\psi\left(u^{*}\right)\right)$ in $L^{2}(\Omega)$ and rewrite as

$$
\eta\left\|M \psi^{i}\right\|^{2}=-\left(u^{i}-u^{*}, M \psi^{i}\right)-\left(\int_{0}^{\tau} k^{*} M \psi^{i} d t, M \psi^{i}\right)+\left(\int_{0}^{\tau}\left(k^{*}-k^{i}\right) M \psi\left(u^{i}\right) d t, M \psi^{i}\right) .
$$

By applying the arguments similar to those in the proofs of (2.25) to the last relation with regard to $(2.22),(2.25)$ and $(2.38)$, we are led to the inequality

$$
\left\|M \psi^{i}\right\|^{2} \leqslant C_{9}^{2}\left(\int_{0}^{t^{*}}\left|k^{*}-k^{i}\right| d t\right)^{2} \exp \left\{\frac{t}{\eta} \max _{t \in\left[0, t^{*}\right]} \delta^{\prime \prime}\right\},
$$

where the positive constant $C_{9}$ depends on $m_{2}, c, C_{1}, C_{5}, C_{6}, \eta, \psi_{0}, T,\left\|U_{0}\right\|,\|f\|,\|\psi(a)\|$ and mes $\Omega$. This proves that $M \psi\left(u^{i}\right) \rightarrow M \psi\left(u^{*}\right)$ in $C\left(\left[0, t^{*}\right] ; L^{2}(\Omega)\right)$ as $i \rightarrow \infty$. Therefore $M \psi\left(u^{*}\right)$ satisfies the estimate (2.25). Moreover, the equation $(2.37)$ suggests that $M\left(\psi\left(u^{i}\right)\right)_{t} \rightarrow$ $M\left(\psi\left(u^{*}\right)\right)_{t}$ in $L^{\infty}\left(\left[0, t^{*}\right] ; L^{2}(\Omega)\right)$ as $i \rightarrow \infty$. By (1.19), this means that $\psi\left(u^{i}\right) \rightarrow \psi\left(u^{*}\right)$ in $C\left(\left[0, t^{*}\right] ; W_{2}^{2}(\Omega)\right)$ and $\left(\psi\left(u^{i}\right)\right)_{t} \rightarrow\left(\psi\left(u^{*}\right)\right)_{t}$ in $L^{\infty}\left(\left[0, t^{*}\right] ; W_{2}^{2}(\Omega)\right)$ as $i \rightarrow \infty$. 
Thus, the existence of the solution to the problem (1.1)-(1.3), (2.8) and, consequently, the inverse problem (1.1)-(1.4) is proved. The uniqueness of the solution follows from the contractibility of the operator $A$. Really, let $\left\{u_{1}, k_{1}\right\}$ and $\left\{u_{2}, k_{2}\right\}$ be two solutions of the problem (1.1)-(1.3), (2.8). Then, by (2.35), the inequality

$$
\left|k_{2}-k_{1}\right|_{\mu}=\left|A k_{2}-A k_{1}\right|_{\mu} \leqslant\left(\frac{\sqrt{2} \max _{t \in[0, T]}\|b\|}{\alpha \psi_{0}^{1 / 2}} \frac{C_{8}}{\mu}+q_{1}\right)\left|k_{2}-k_{1}\right|_{\mu},
$$

holds, which suggests in view of $(2.36)$ that $\left|k_{2}-k_{1}\right|_{\mu} \leqslant 0$, i. e. $k_{1}=k_{2}$ on $\left[0, t^{*}\right]$. This along with (2.31) for $\left\{u_{1}-u_{2}, k_{1}-k_{2}\right\}$ provides in turn that $u_{1}-u_{2}=0$ on $\left[0, t^{*}\right]$. Theorem is proved.

It may appear that the hypotheses of Theorem 2.1 are unwieldy and suspicious. However the following example confirms that there are physically meaningful input data satisfying these hypotheses.

Let us consider the nonlinear pseudoparabolic equation of filtration in the fissured rock [2].

$$
\left(u_{t}+\varepsilon \mu B_{u}(u)\right)_{t}+B_{u}(\zeta(u))=0,
$$

where $u$ is the pressure of a liquid in pores, $B_{u}=-(1 / \mu) \operatorname{div}(l(u) \nabla)$ and $\mu$ is the viscosity of the liquid. We can account for the weakly compressible liquid by setting $\zeta(u)=k(t) u$ where the coefficient $k(t)$ characterized the hydraulic properties of the fissured medium. In this case, we are led to the equation (1.1) where $M=-\Delta, \eta=\varepsilon \mu, \psi(u)=\int_{0}^{u} l(z) d z, f \equiv 0$. The permeability $l(u)$ is a continuous nonincreasing positive function of the pressure. Under such suppositions the operator $M$ and the function $\psi(\rho)$ satisfy the assumptions I-IV.

The steady-state initial condition [1] is appropriate to the condition (1.1) with $U_{0}=a(0, x)$. In the conditions (1.3) and (1.4) we set $\beta(t, x)=\psi^{-1}\left(\sum_{j=1}^{3} a_{j} x_{j}+g_{a}(t)\right), \omega(t, x)=\sum_{j=1}^{3} b_{j} x_{j}+g_{b}(t)$ and $\varphi_{1} \equiv 0$. We suppose that $\Omega \subset R_{+}^{3}=\left\{x \mid x \in \mathbf{R}^{3}, x_{i} \geqslant 0, i=1,2,3\right\}, g_{a}(0)>0, g_{b}(t)>0$, $g_{a}^{\prime}(t) \geqslant 0, b_{i}(t) \geqslant 0$ on $[0, T]$ and $a_{i}, b_{i} \geqslant 0, i=1,2,3$. Let also $\sum_{j=1}^{3} a_{j} b_{j} \geqslant \alpha>0$. Then the conditions (2.2)-(2.4), (2.6) are fulfilled. If $\varphi_{2}(t) \geqslant \varphi_{0}>0$ and the domain $\Omega$ is sufficiently small, then the inequality $(2.5)$ holds with some $0<\Phi_{0}<\varphi_{0}$.

This research was supported by the Government of the Russian Federation, grant 14. Y26.31.0006.

\section{References}

[1] G.I.Barenblatt, Iu.P.Zheltov, I.N.Kochina, Basic concepts in the theory of seepage of homogeneous liquids in fissured blocks [strata], J. Appl. Math. Mech., 24(1960), 1286-1303 (in Russian).

[2] M.Bohm, R.E.Showalter, Diffusion in Fissured Media, SIAM J. of Mathematical Anal., 16(1985), 500-509.

[3] H.Gajewski, K.Gröger, K.Zacharias, Nichtlinear Operatorgleichungen und Operatordifferentialgleichungen. Mathematische Lehrbücher und Monographien, II. Abteilung, Mathematische Monographien, Akademie-Verlag, Berlin, Vol. 38, 1974.

[4] A.I.Kozhanov, O razreshimosti obratnykh zadach koeffitsientov dlya nekotorikh uravnenii sobolevskogo tipa (On the solvability of the coefficient inverse problems for equations of Sobolev type), Nauchniye vedomosti Belgorodskogo gosudarstvennogo universiteta. Seriya "Matematika. Phizika", 5(2010), 88-98 (in Russian). 
[5] J.-L.Lions, E.Magenes, Problemes aux Limites Non Homogenes et Applications, Vol. 1, Travaux et Recherches Mathematiques, no. 17, Dunod, Paris, 1968.

[6] A.Sh.Lyubanova, On an inverse problem for quasi-linear elliptic equation, Journal of Siberian Federal University. Mathematics and Physics, 8(2015), 38-48.

[7] A.Sh.Lyubanova, A.Tani, An inverse problem for pseudoparabolic equation of filtration. The existence, uniqueness and regularity, Appl. Anal., 90(2011), 1557-1571.

[8] M.Sh.Mamayusupov, O zadache opredeleniya koeffitsiyentov psevdoparabolicheskogo uravneniya (The problem of determining coefficients of a pseudoparabolic equation), Studies in integro-differential equations, Ilim, Frunze, 1983, no. 16, 290-297 in (Russian).

[9] W.Rundell, Determination of an unknown nonhomogeneous term in a linear partial differential equation from overspecified boundary data, Appl. Anal., 10(1980), 231-242.

[10] R.E.Showalter, T.W.Ting, Pseudoparabolic partial differential equations, SIAM J. Math. Anal., 1(1970), 1-26.

[11] A.G.Sveshnikov, A.B.Alshin, M.O.Korpusov, Yu.D.Pletner, Lineyniye i nelineyniye uravneniya sobolevskogo tipa (Linear and nonlinear equations of the Sobolev type), Physmatlit, Moscow, 2007 (in Russian).

\section{Обратная задача для нелинейного псевдопараболического уравнения типа фильтрации}

Анна Ш. Любанова

Институт космических и информационных технологий Сибирский федеральный университет Киренского, 26, Красноярск, 660074

Россия

В статъе обсуждается корректность обратной задачи отыскания неизвестного коэббициента, зависящего от времени в нелинейном псевдопараболическом уравнении третъего порядка по дополнительной информащии о решении на гранище. Доказывается теорема существования и единственности сильного решения. При доказательстве теоремы исходная обратная задача сводится $\kappa$ эквивалентной задаче с операторным уравнением для неизвестного коэффициента.

Ключевые слова: локальная теорема существования и единственности, априорная оценка, обратная задача, нелинейное уравнение высшего порядка, псевдопараболическое уравнение, фильтрация. 